\title{
PROBLEMS ON AVERAGES AND LACUNARY MAXIMAL FUNCTIONS
}

\author{
ANDREAS SEEGER AND JAMES WRIGHT
}

\begin{abstract}
We prove three results concerning convolution operators and lacunary maximal functions associated to dilates of measures. First we obtain an $H^{1}$ to $L^{1, \infty}$ bound for lacunary maximal operators under a dimensional assumption on the underlying measure and an assumption on an $L^{p}$ regularity bound for some $p>1$. Secondly, we obtain a necessary and sufficient condition for $L^{2}$ boundedness of lacunary maximal operator associated to averages over convex curves in the plane. Finally we prove an $L^{p}$ regularity result for such averages. We formulate various open problems.
\end{abstract}

\section{INTRODUCTION}

We consider a compactly supported finite Borel measure $\mu$ and define its dyadic dilates by $\left\langle\mu_{k}, f\right\rangle=\left\langle\mu, f\left(2^{k} \cdot\right)\right\rangle$. The main objects of this paper are the convolutions $f \mapsto f * \mu_{k}$ and the lacunary maximal function given by

$$
\mathcal{M} f(x)=\sup _{k \in \mathbb{Z}}\left|f * \mu_{k}(x)\right| .
$$

Throughout this paper the dilates $2^{k}$ can be replaced by more general lacunary dilates $\lambda_{k}$ satisfying $\inf _{k} \lambda_{k+1} / \lambda_{k}>1$.

If $\mu$ satisfies the condition $\widehat{\mu}(\xi)=O\left(|\xi|^{-\varepsilon}\right.$ ) (some $\varepsilon>0$ ), then $L^{p_{\text {- }}}$ boundedness of $\mathcal{M}$ holds in the range $1<p<\infty$, see e.g. [9]. For suitable classes of examples we discuss two problems, namely what may happen in the limiting case $p=1$, and, secondly, what could be said about boundedness of $\mathcal{M}$ if the above decay condition on $\widehat{\mu}$ is relaxed.

Notation: For two nonnegative quantities $A$ and $B$ let $A \lesssim B$ denote the statement that $A \leq C B$ for some constant $C$. The Lebesgue measure of a set $E$ is denoted by meas $(E)$. Throughout we work with a fixed inhomogeneous dyadic frequency decomposition $\left\{P_{k}\right\}_{k=0}^{\infty}$. Let $\beta_{\circ} \in C_{c}^{\infty}(\mathbb{R})$ be supported in $(-1,1)$ and equal to 1 in $[-1 / 2,1 / 2]$. Define the operators $P_{k}$ by $\widehat{P_{0} f}(\xi)=$ $\beta_{\circ}(|\xi|) \widehat{f}(\xi)$ if $k=0$ and by $\widehat{P_{k} f}(\xi)=\left(\beta_{\circ}\left(2^{-k}|\xi|\right)-\beta_{\circ}\left(2^{-k+1}|\xi|\right)\right) \widehat{f}(\xi)$ if $k>0$.

1991 Mathematics Subject Classification. 42B25 (primary), 42B15, $42 \mathrm{~B} 30$.

Key words and phrases. Regularity of averages, Lacunary maximal functions.

A.S. supported in part by NSF 0652890 and MEC grant MTM2010-16518. J.W. supported in part by an EPSRC grant. 
$\mathbf{H}^{\mathbf{1}} \rightarrow \mathbf{L}^{\mathbf{1}, \infty}$ boundedness of lacunary maximal operators. Concerning the case $p=1, \mathcal{M}$ can never be bounded on $L^{1}\left(\mathbb{R}^{d}\right)$, outwith the trivial case. One can ask whether the smoothing condition $\widehat{\mu}(\xi)=O\left(|\xi|^{-\varepsilon}\right)$ implies that $\mathcal{M}$ is of weak-type $(1,1)$, i.e. maps $L^{1}\left(\mathbb{R}^{d}\right)$ to the Lorentz space $L^{1, \infty}\left(\mathbb{R}^{d}\right)$. To the best of our knowledge no counterexample and no example is known for the case that $\mu$ is a singular measure with the decay assumption on $\widehat{\mu}$.

For various classes of singular measures it has been observed that a somewhat weaker endpoint inequality holds, namely that $\mathcal{M}$ maps the (usual isotropic) Hardy space $H^{1}\left(\mathbb{R}^{d}\right)$ to $L^{1, \infty}\left(\mathbb{R}^{d}\right)$. The first results in this direction are due to Christ [5] who used some powerful variants of CalderónZygmund theory. We formulate a theorem which unifies and extends some previous results, with some simplification in the proofs. In what follows we let $\mathcal{A}$ denote the convolution operator $\mathcal{A}: f \mapsto f * \mu$.

Theorem 1.1. For $\rho>0$ let $\mathcal{N}_{\Sigma}(\rho)$ be the minimal number of balls of radius $\rho$ needed to cover the support of $\mu$. Let $0<s \leq d$ and suppose that

$$
\begin{aligned}
& \sup _{0<\rho<1} \rho^{d-s} \mathcal{N}_{\Sigma}(\rho)<\infty \\
& \sup _{k>0} 2^{k(d-s)\left(1-\frac{1}{p}\right)}\left\|P_{k} \mathcal{A}\right\|_{L^{p}\left(\mathbb{R}^{d}\right) \rightarrow L^{p}\left(\mathbb{R}^{d}\right)}<\infty, \quad \text { for some } p>1 .
\end{aligned}
$$

Then the lacunary maximal operator $\mathcal{M}$ maps $H^{1}\left(\mathbb{R}^{d}\right)$ into $L^{1, \infty}\left(\mathbb{R}^{d}\right)$.

The covering condition (11) is a dimensional assumption on the support of the measure; in particular when $s$ is an integer it is satisfied if $\operatorname{supp}(\mu)$ is contained in an imbedded manifold of codimension $s$. The assumption (2) expresses an optimal smoothing result in the category of Besov spaces, for $p$ near 1. We note that assumptions (11) together with assumption (21) imply stronger regularity results in Sobolev and Triebel-Lizorkin spaces, see [23. When $p=2$ the assumption (2) is equivalent with the inequality $\widehat{\mu}(\xi)=O\left(|\xi|^{-\frac{d-s}{2}}\right)$. In this case we recover results by Oberlin [18] and Heo [13], both extending Christ's original result and method of proof in [5].

Examples. (i) Our main new example concerns the case of arclength measure on a compact curve in $\mathbb{R}^{3}$ with nonvanishing curvature and torsion. Here $d=3, s=2$ and the main regularity assumption is a recent result of Pramanik and one of the authors [24], established for $p<\left(p_{W}+2\right) / p_{W}$ where $p_{W}<\infty$ is an exponent for which a deep inequality of T. Wolff [34] on decompositions of cone multipliers holds.

(ii) As in 29, 14] one can consider hypersurfaces on which the Gaussian curvature does not vanish to infinite order. Then of course (11) holds with $s=1$ and, by a result of Sogge and Stein [30, inequality (2) holds for some values of $p>1$ (in a range depending on the order of vanishing of the curvature). 
(iii) An interesting example occurs in [15] where $\Sigma$ is a portion of the light cone in $\mathbb{R}^{d}$ when $d \geq 5$. In this case one can see condition (2) as a consequence of a sharp space time inequality for spherical means, see inequality (10.9) in [16].

Open problems. There are many, concerning both the hypotheses and the conclusion of Theorem 1.1.

(a) Given the dimensionality assumption (11), under what conditions does (2) hold? More concretely, if $d \mu=\chi d \sigma$ and $d \sigma$ is arclength measure on (a compact piece of) a curve of finite type in $\mathbb{R}^{d}$, does (2) hold with $s=d-1$ and some $p>1$. This is open in dimensions $d \geq 4$. Similarly, is $\mathcal{M}: H^{1} \rightarrow L^{1, \infty}$ for these examples?

(b) More generally, if $\Sigma$ is a manifold of finite type (in the sense of ch. VIII, §3.2. of [31]) does it follow that the lacunary maximal operator maps $H^{1}$ to $L^{1, \infty}$ ? See 33 for a measure supported on a cylinder for which the associated lacunary maximal operator which does map $H^{1}$ to $L^{1, \infty}$ but for which the hypothesis (2) does not hold.

(c) In Theorem 1.1, can one replace the usual isotropic dilations by nonisotropic ones, with the corresponding change of Hardy spaces, but keeping the isotropic assumption (2)? See [5] for results on averaging operators along curves in two dimensions and related results in [29], [14] for hypersurfaces. The question whether the 'maximal function along the $\left(t, t^{2}, t^{3}\right)$ maps the corresponding anisotropic Hardy space $\mathcal{H}^{1}$ to $L^{1, \infty}$ is currently open.

(d) In [29] it was shown that the lacunary maximal operators associated to hypersurfaces of finite type (with respect to an arbitrary dilation group) are of weak type $L \log \log L$. This is the current result closest to a perhaps conjectured weak type $L^{1}$ bound. It is open whether one can prove a similar result merely under the regularity assumption (2).

Remark. We take this opportunity to mention a fallacious argument in the exceptional set estimate in $\S 5$ of the article [29] and thank Neal Bez for pointing it out. A correction is posted on one of the authors' website (see the reference to [29] below).

Lacunary maximal operators associated to convex curves in the plane. Let $\Omega$ be a convex open domain in the plane with compact closure so that the origin is contained in $\Omega$. We let $\sigma$ be the arclength measure on the boundary $\partial \Omega$ and consider the question of $L^{p}$ boundedness of the lacunary maximal operator associated to $\partial \Omega$,

$$
\mathcal{M} f(x)=\sup _{k \in \mathbb{Z}}\left|\int_{\partial \Omega} f\left(x-2^{k} y\right) d \sigma(y)\right| .
$$

As mentioned above, if $|\widehat{\sigma}(\xi)|=O\left(|\xi|^{-\varepsilon}\right)$ for some $\varepsilon>0$, then $\mathcal{M}$ is $L^{p}$ bounded for $1<p<\infty$. Now we are aiming for much weaker hypotheses. 
The decay of $\widehat{\sigma}$ is strongly related to a geometric quantity. Given a unit vector $\theta$ let $\ell^{+}(\theta)$ be the unique supporting line with $\theta$ an outer normal to $\partial \Omega$, i.e. the affine line perpendicular to $\theta$ which intersects $\partial \Omega$ so that $\Omega$ is a subset of the halfspace $\left\{x: x=y-t \theta: t>0, y \in \ell^{+}(\theta)\right\}$. Similarly define $\ell^{-}(\theta)$ as the unique affine line perpendicular to $\theta$ which intersects $\partial \Omega$ and $\Omega$ is a subset of the halfspace $\left\{x: x=y+t \theta: t>0, y \in \ell^{-}(\theta)\right\}$. For small $\delta>0$ define the $\operatorname{arcs}($ or 'caps')

$$
\mathcal{C}^{ \pm}(\theta, \delta)=\left\{y \in \partial \Omega: \operatorname{dist}\left(y, \ell^{ \pm}\right) \leq \delta\right\} .
$$

By a compactness consideration it is easy to see that there is $\delta_{0}>0$ so that for all $\theta \in S^{1}$ and all $\delta<\delta_{0}$ the $\operatorname{arcs} \mathcal{C}^{+}(\theta, \delta)$ and $\mathcal{C}^{-}(\theta, \delta)$ are disjoint. Let, $\Lambda(\theta, \delta)$ be the maximum of the length of these caps:

$$
\Lambda(\theta, \delta)=\max _{ \pm} \sigma\left(\mathcal{C}^{ \pm}(\theta, \delta)\right) .
$$

The analytic significance of this quantity is that it gives a very good estimate for the size of Fourier transform $\widehat{\sigma}$, namely for every $\theta \in S^{1}$ and $R \geq 1$

$$
|\widehat{\sigma}(R \theta)| \leq C_{\Omega} \Lambda\left(\theta, R^{-1}\right) .
$$

This is shown in [1] under the hypothesis for convex domains in the plane with smooth boundary, with no quantitative assumption on the second derivative. The general case follows by a simple approximation procedure (see also [21], 22] for similar observations).

We note that for classes of multipliers satisfying standard symbol assumptions one can insure boundedness under rather weak decay assumptions on the symbol, but it is not clear what the optimal conditions are; moreover the usual method of square-functions is often not the appropriate tool ( $c f$. [7], [12]). In this light the following characterization for $p=2$ in terms of the quantities $\Lambda(\theta, \delta)$ is perhaps surprising (as well as the fact that it can be proved using simple square-function arguments).

Theorem 1.2. The operator $\mathcal{M}$ is bounded on $L^{2}\left(\mathbb{R}^{2}\right)$ if and only if

$$
\sup _{\theta \in S^{1}} \int_{0}^{\delta_{0}} \Lambda(\theta, \delta)^{2} \frac{d \delta}{\delta}<\infty
$$

Problem: For $q \neq 2$, find necessary and sufficient conditions on $\partial \Omega$ in order for $\mathcal{M}$ to be bounded on $L^{q}\left(\mathbb{R}^{2}\right)$.

It may be interesting to look at specific 'flat' examples. By testing $\mathcal{M}$ on functions supported in thin strips we shall obtain a necessary condition

$$
\sup _{\|f\|_{q}=1}\|\mathcal{M} f\|_{q} \geq c \sup _{\theta \in S^{1}}\left(\int_{0}^{\delta_{0}} \Lambda(\theta, \delta)^{q} \frac{d \delta}{\delta}\right)^{1 / q}
$$

for all $q$. We note that if $\partial \Omega$ has only one "flat" point near which $\partial \Omega$ can be parametrized as the graph of $C+\exp \left(-1 /|t|^{a}\right)$ with $C \neq 0$, then this $L^{q}$ condition holds iff $a<q$. Thus in this case $L^{2}$ boundedness of $\mathcal{M}$ holds if and only if $a<2$. 
$\mathbf{L}^{\mathbf{p}}$-regularity of averages. As in the previous section we consider convex curves $\Sigma$, say boundaries of a convex domain but the position of the origin will not play a role now. The estimate (44) can be interpreted as an $L^{2}$ regularity result for the integral operator $\mathcal{A}: f \mapsto f * \sigma$. We reformulate this with the standard dyadic frequency decomposition $\left\{P_{k}\right\}_{k=0}^{\infty}$ as above. Then setting

$$
\omega_{k}=\frac{1}{\sup _{\theta \in S^{1}} \Lambda\left(\theta, 2^{-k}\right)}
$$

(41) says that the inequality

$$
\left\|\left(\sum_{k>0} \omega_{k}^{2}\left|P_{k} \mathcal{A} f\right|^{2}\right)^{1 / 2}\right\|_{p} \lesssim\|f\|_{p}
$$

holds for $p=2$.

We are now interested in analogous $L^{p}$ regularity results, i.e. we wish to determine the range of $p$ for which (8) holds, with the optimal weight $\omega_{k}$ in (77). In case the curvature vanishes somewhere one expects this inequality to hold for some $p \neq 2$; for example if $\Sigma$ is of finite type, and if $m$ is the order of maximal contact of tangent lines with $\Sigma$ then (8) holds with the optimal $\omega_{k}=2^{-k / m}$ for the range $\frac{m}{m-1}<p<m$, see [32, [6] (and also [26], 35. for variable coefficient analogues). Given these known examples we are mainly interested in very flat cases. We shall prove (8) for a family of curves with additional hypotheses which cover interesting examples for which the curvature vanishes of infinite order at a point. In those flat cases one gets (8) in the full range $1<p<\infty$.

After a localization we assume that (part of) the convex curve is given as a graph $(t, \gamma(t))$ for $0 \leq t \leq 1$ and consider the integral operator

$$
A f(x)=\int_{0}^{1} f\left(x_{1}-t, x_{2}-\gamma(t)\right) d t .
$$

Theorem 1.3. Let $\gamma$ be of class $C^{3}$ on $(0,1]$ and of class $C^{2}$ on $[0,1]$. Assume that $\gamma(0)=\gamma^{\prime}(0)=\gamma^{\prime \prime}(0)=0, \gamma^{\prime \prime}$ is nonnegative and strictly increasing on $[0,1]$, and furthermore, the limit

$$
b=\lim _{t \rightarrow 0+} \frac{\gamma^{\prime \prime}(t)}{t \gamma^{\prime \prime \prime}(t)}
$$

exists with $b \in[0, \infty)$. Let

$$
w_{k}=\frac{1}{\gamma^{-1}\left(2^{-k}\right)} \text {. }
$$

Then the inequality

$$
\left\|\left(\sum_{k>0} w_{k}^{2}\left|P_{k} A f\right|^{2}\right)^{1 / 2}\right\|_{p} \lesssim\|f\|_{p}
$$

holds for $1+\frac{b}{1+b}<p<2+b^{-1}$ (and thus for $1<p<\infty$ if $b=0$ ). 
Examples. We note that, in the setup of this theorem, $w_{k} \approx \omega_{k}$ as defined in (17). In the case $\gamma_{\circ}(t)=t^{m}$ with $m>2$ we recover the known result mentioned above since $b^{-1}=m-2$ and $w_{k} \approx 2^{-k / m}$. In the flat case $\gamma_{1}(t)=e^{-t^{-a}}$ we have $b=0$ and $w_{k} \approx k^{-1 / a}$. We may consider evem flatter cases: Let $\exp _{*}^{n}$ the $n$-fold iteration $\exp \circ \cdots \circ \exp$ and $\log _{*}^{n}$ the $n$-fold iteration of ln. For large $C>0$ consider $\gamma_{2}(t)=\exp \left(-\exp _{*}^{n}\left(C t^{-\lambda}\right)\right)$. Then $b=0$ and $w_{k} \approx\left(\log _{*}^{n}\left(e^{n}+k\right)\right)^{-1 / \lambda}$.

Open problems.

(i) For the curves $\Gamma(t)=(t, \gamma(t))$ featured in Theorem 1.3 let $\mathfrak{M}$ be the maximal function along $\Gamma$ (as in (27) below). Does $\mathfrak{M}$ map the Hardy-space $H_{\text {prod }}^{1}\left(\right.$ associated with the two-parameter dilations $\left.\left(t_{1} \cdot, t_{2} \cdot\right)\right)$ to the Lorentz space $L^{1,2}$ ? Similar questions can be formulated for certain singular integrals along $\Gamma$. For the finite type case $\left(\gamma(t)=t^{m}\right)$ such estimates can be found in [27]. For the flat cases one would need to further explore Hardy space structures associated to the curve $\Gamma$.

(ii) Let $\mathcal{D}^{1 / m} f=\mathcal{F}^{-1}\left[\left(1+|\xi|^{2}\right)^{1 / 2 m} \widehat{f}\right]$, the fractional Bessel derivative of order $1 / m$. For the finite type $m$ case there are endpoint estimates involving Lorentz-spaces $L^{p, 2}$, namely it was shown in [27] that $\mathcal{D}^{1 / m} A$ maps $L^{m, 2}$ to $L^{m}$ and $L^{m^{\prime}, 2}$ to $L^{m^{\prime}}$, see 6 for the sharpness of such results. It would be interesting to investigate sharp regularity results for general $\gamma$. One aims to bound the square-function $\left(\sum_{k>0} w_{k}^{2}\left|P_{k} A f\right|^{2}\right)^{1 / 2}$ in natural Orlicz or Orlicz-Lorentz spaces associated with $\gamma$. As suggested by the method in [27], endpoint results should be related to a resolution of problem (i).

(iii) Can one prove (81) for more general convex domains; in particular, can one relax the monotonicity assumption on $\gamma^{\prime \prime}$ in Theorem 1.3.

This paper. The proof of Theorem 1.1 will be given in $\$ 2$, The proof of Theorem 1.2 is in 93 . The proof of Theorem 1.3 is in in 34 In 95 we formulate yet another problem concerning lacunary maximal functions for dilates of a simple Marcinkiewicz multiplier.

Acknowledgements: We would like to thank Terry Tao for conversations on the subject of lacunary maximal operators and collaboration on [28] and [29].

\section{Proof of theorem 1.1}

Atomic decompositions. In what follows $\psi$ will denote a nontrivial $C^{\infty}$ function with compact support in $\{x:|x| \leq 1 / 2\}$ so that $\widehat{\psi}(\xi) \neq 0$ for $\frac{1}{4} \leq$ $|\xi| \leq 4$ and $\widehat{\psi}$ vanishes to order $10 d$ at the origin. We let $\psi_{l}=2^{-l d} \psi\left(2^{-l}\right.$.).

We use the atomic decomposition based on a square-function characterization as given in [4]; for variants and applications of this method see [25], 
[28], [16]. Given $f$ in $H^{1}$ one can write $f=\sum_{Q} b_{Q}$ where this sum converges in $H^{1}$, each $b_{Q}$ is supported in the double of the dyadic cube $Q$ with sidelength $2^{L(Q)}$, and has the following fine structure. We have

$$
b_{Q}=\sum_{j \leq L(Q)} \psi_{j} * \psi_{j} * b_{Q, j}
$$

where $b_{Q, j}$ can be decomposed as $b_{Q, j}=\sum_{R \in \Re(Q, j)} e_{R}$, the families $\Re(Q, j)$ consist of dyadic cubes $R$ of sidelength $2^{j}$ contained in $Q$ with disjoint interior, the bounded function $e_{R}$ is supported on $R$, and finally

$$
\sum_{Q}|Q|^{1 / 2}\left(\sum_{j \leq L(Q)} \sum_{R \in \mathfrak{R}(Q, j)}\left\|e_{R}\right\|_{2}^{2}\right)^{1 / 2} \lesssim\|f\|_{H_{1}} .
$$

We set

$$
\gamma_{Q, j}=\left(\sum_{R \in \Re(Q, j)}\left\|e_{R}\right\|_{2}^{2}\right)^{1 / 2}, \quad \gamma_{Q}=\left(\sum_{j \leq L(Q)} \gamma_{Q, j}^{2}\right)^{1 / 2},
$$

and note that

$$
\begin{aligned}
\left\|b_{Q, j}\right\|_{2} & \lesssim \gamma_{Q, j}, \\
\left\|b_{Q}\right\|_{2} & \lesssim \gamma_{Q},
\end{aligned}
$$

and

$$
\sum_{Q}|Q|^{1 / 2} \gamma_{Q} \lesssim\|f\|_{H^{1}}
$$

The weak type inequality. The condition in (2) becomes more restrictive as $p$ increases and therefore we may assume $p \leq 2$.

By a scaling argument we may assume that $\mu_{0}=\mu$ is supported in the unit ball centered at the origin. (the operator norm will depend on that scaling).

We need to show that

$$
\operatorname{meas}\left(\left\{x: \sup _{k}\left|\mu_{k} * \sum_{Q} b_{Q}\right|>\alpha\right\}\right) \lesssim \alpha^{-1}\|f\|_{H^{1}} .
$$

To achieve this we assign for each $Q$ an integer $\tau(Q)$ depending on $\alpha$, defined as follows. We first let $\widetilde{\tau}(Q)$ be the smallest integer $\tau$ for which

$$
2^{(d-s) \tau} 2^{s L(Q)} \geq \alpha^{-1}|Q|^{1 / 2} \gamma_{Q}
$$

(or $-\infty$ if there is no such smallest integer) and define

$$
\tau(Q)=\max \{L(Q), \widetilde{\tau}(Q)\} .
$$

We form an exceptional set depending on $\alpha$ by

$$
\mathcal{E}=\bigcup_{Q} \bigcup_{k: L(Q)<k \leq \tau(Q)}\left(\operatorname{supp}\left(\mu_{k}\right)+Q^{*}\right)
$$


where $Q^{*}$ is the tenfold dilate of $Q$ with respect to its center. Note that $\left(\operatorname{supp} \mu_{k}+Q^{*}\right)$ is contained in the $2^{k}$-dilate of a $C 2^{L(Q)-k}$ neighborhood of $\operatorname{supp}(\mu)$. By the assumption on $\mu$ this neighborhood can be covered with $\lesssim 2^{(k-L(Q))(d-s)}$ balls of radius $2^{L(Q)-k}$. Thus

$$
\operatorname{meas}\left(\operatorname{supp}\left(\mu_{k}\right)+Q^{*}\right) \leq C_{1} 2^{k d} 2^{(k-L(Q))(d-s)} 2^{(L(Q)-k) d}=C_{1} 2^{k(d-s)} 2^{L(Q) s}
$$

and thus

$$
\operatorname{meas}(\mathcal{E}) \lesssim \sum_{Q: L(Q)<\tau(Q)} 2^{\tau(Q)(d-s)} 2^{L(Q) s}
$$

By the minimality property of $\tau(Q)$ in its definition it follows that for the case $L(Q)<\tau(Q)$ the inequality $2^{(\tau(Q)-1)(d-s)} 2^{L(Q) s} \leq \alpha^{-1}|Q|^{1 / 2} \gamma_{Q}$ is satisfied. Thus

$$
\operatorname{meas}(\mathcal{E}) \lesssim \sum_{Q: L(Q)<\tau(Q)} \alpha^{-1}|Q|^{1 / 2} \gamma_{Q} \lesssim \alpha^{-1}\|f\|_{H^{1}}
$$

We $\operatorname{split}_{\sup _{k}}\left|f * \mu_{k}(x)\right|$ into three parts depending on $\alpha$.

$$
\begin{gathered}
I(x)=\sup _{k}\left|\mu_{k} * \sum_{Q: \tau(Q)<k} b_{Q}\right|, \\
I I(x)=\sup _{k}\left|\mu_{k} * \sum_{Q: L(Q)<k \leq \tau(Q)} b_{Q}\right|, \\
I I I(x)=\sup _{k}\left|\mu_{k} * \sum_{Q: k \leq L(Q)} b_{Q}\right| .
\end{gathered}
$$

Note that $I I$ is supported in $\mathcal{E}$ and thus

$$
\operatorname{meas}\left(\left\{x: \sup _{k}\left|\mu_{k} * \sum_{Q} b_{Q}\right|>\alpha\right\}\right) \lesssim \frac{\|I\|_{p}^{p}}{\alpha^{p}}+\operatorname{meas}(\mathcal{E})+\frac{\|I I I\|_{1}}{\alpha}
$$

where $p$ is as in assumption (2).

The estimation for $\|I I I\|_{1}$ is straightforward and just uses the $L^{2}$ boundedness of the lacunary maximal operator. Note that for $k \leq L(Q)$ the function $\mu_{k} * f$ is supported in $Q^{*}$. We estimate

$$
\begin{aligned}
\|I I I\|_{1} & \leq \sum_{Q}\left\|\sup _{k \leq L(Q)}\left|\mu_{k} * b_{Q}\right|\right\|_{1} \\
& \leq \sum_{Q}\left|Q^{*}\right|^{1 / 2}\left\|\mathcal{M} b_{Q}\right\|_{2} \\
& \lesssim \sum_{Q}|Q|^{1 / 2}\left\|b_{Q}\right\|_{2} \lesssim\|f\|_{H^{1}}
\end{aligned}
$$


We turn to the main term $I$ and estimate

$$
\begin{aligned}
\|I\|_{p}^{p} & =\left\|\sup _{k}\left|\mu_{k} * \sum_{L} \sum_{\substack{Q: L(Q)=L \\
\tau(Q) \leq k}} \sum_{n \geq 0} \psi_{L-n} * \psi_{L-n} * b_{Q, L-n}\right|\right\|_{p} \\
& \leq\left(\sum_{n=0}\left(\sum_{k}\left\|I_{n, k}\right\|_{p}^{p}\right)^{1 / p}\right)^{p}
\end{aligned}
$$

where

$$
I_{n, k}=\mu_{k} * \sum_{L} \sum_{\substack{Q: L(Q)=L \\ \tau(Q) \leq k}} \psi_{L-n} * \psi_{L-n} * b_{Q, L-n} .
$$

We shall bound $\sum_{k}\left\|I_{n, k}\right\|_{p}^{p}$ with some exponential gain in $n$.

We first observe as a consequence of assumption (2) the simple convolution inequality $\left\|\mu * \psi_{j} * g\right\|_{p} \lesssim 2^{-j(d-s) / p^{\prime}}\|g\|_{p}$. Indeed this follows from (2) by observing that $\left\|P_{k} \psi_{j}\right\|_{1} \lesssim 2^{-|j-k| 2 d}$ where we use the vanishing moment assumption on $\psi_{j}$. By scaling, the operator of convolution with $\mu_{k} * \psi_{L-n}$ has the same operator norm as the operator of convolution with $\mu_{0} * \psi_{L-n-k}$ which is $O\left(2^{-(k+n-L)(d-s) / p^{\prime}}\right)$. Because of the almost orthogonality of the $\psi_{j}$ we may apply the inequality $\left\|\sum \psi_{j} * g_{j}\right\|_{p} \lesssim\left(\sum_{j}\left\|g_{j}\right\|_{p}^{p}\right)^{1 / p}$. Thus we get

$$
\begin{aligned}
\left\|I_{n, k}\right\|_{p} & \lesssim\left(\sum_{L}\left\|\mu_{k} * \psi_{L-n} * \sum_{\substack{Q: L(Q)=L \\
\tau(Q) \leq k}} b_{Q, L-n}\right\|_{p}^{p}\right)^{1 / p} \\
& \lesssim 2^{-(k+n-L)(d-s) / p^{\prime}}\left(\sum_{L}\left\|\sum_{\substack{Q: L(Q)=L \\
\tau(Q) \leq k}} b_{Q, L-n}\right\|_{p}^{p}\right)^{1 / p} \\
& \lesssim 2^{-(k+n)(d-s) / p^{\prime}}\left(\sum_{Q: \tau(Q) \leq k} 2^{L(Q)(d-s)(p-1)}\left\|b_{Q, L-n}\right\|_{p}^{p}\right)^{1 / p},
\end{aligned}
$$

where the last inequality follows from the disjointness of the $Q$ with fixed $L=L(Q)$. Thus we get after interchanging summations and summing in $k \geq \tau(Q)$

$$
\begin{aligned}
\left(\sum_{k}\left\|I_{n, k}\right\|_{p}^{p}\right)^{1 / p} & \lesssim 2^{-n(d-s) / p^{\prime}}\left(\sum_{Q} 2^{(L(Q)-\tau(Q))(d-s)(p-1)}\left\|b_{Q, L-n}\right\|_{p}^{p}\right)^{1 / p} \\
& \lesssim 2^{-n(d-s) / p^{\prime}}\left(\sum_{Q} 2^{(L(Q)-\tau(Q))(d-s)(p-1)}|Q|^{1-p / 2} \gamma_{Q}^{p}\right)^{1 / p}
\end{aligned}
$$

In the last estimate we have used Hölder's inequality and $\gamma_{Q, j} \leq \gamma_{Q}$. By the definition of $\tau(Q)$ we have

$$
2^{-(d-s) \tau(Q)} \leq 2^{-s L(Q)} \alpha|Q|^{-1 / 2} \gamma_{Q}^{-1},
$$


no matter whether $\tau(Q)>L(Q)$ or $\tau(Q)=L(Q)$. This leads to

$$
2^{(L(Q)-\tau(Q))(d-s)(p-1)}|Q|^{1-p / 2} \gamma_{Q}^{p} \leq \alpha^{p-1}|Q|^{1 / 2} \gamma_{Q}
$$

and consequently we get

$$
\sum_{k}\left\|I_{n, k}\right\|_{p}^{p} \leq C^{p} 2^{-n(d-s)(p-1)} \alpha^{p-1} \sum_{Q}|Q|^{1 / 2} \gamma_{Q}
$$

which by (15) implies

$$
\|I\|_{p}^{p} \leq \widetilde{C}^{p} \alpha^{p-1} \sum_{Q}|Q|^{1 / 2} \gamma_{Q} .
$$

We finish the proof of (11) by combining (13), (12), (14) and (16).

\section{Proof of Theorem 1.2}

We shall first prove the necessary condition (6) for $L^{q}$-boundedness of $\mathcal{M}$. We check the lower bound by providing an example for $\theta=e_{2}$ and the general case follows by rotating the curve. From the positivity of $\mathcal{M}$ and translation invariance we may reduce to the case where $\mathcal{M}$ is replaced by the maximal operator

$$
\begin{aligned}
& M f(x)=\sup _{k \in \mathbb{Z}}\left|A_{k} f(x)\right| \\
& \text { with } A_{k} f(x):=\left|\int_{|t| \leq \epsilon} f\left(x_{1}-2^{k} t, x_{2}-2^{k} L+2^{k} \gamma(t)\right) d t\right|
\end{aligned}
$$

here $t \mapsto \gamma(t)$ is a convex function with $\gamma(0)=0, L>0, \varepsilon>0$ and the line $\left\{x_{2}=L\right\}$ is a supporting line at $(0, L)$.

We test $M$ on the functions

$$
f_{\eta}(x)=(4 \eta)^{-1 / q} \chi_{E_{\eta}}(x) \text { where } E_{\eta}:=\left\{x \in \mathbb{R}^{2}:\left|x_{1}\right| \leq 1,\left|x_{2}\right| \leq \eta\right\}
$$

for small $\eta>0$. Then $\left\|f_{\eta}\right\|_{q}=1$. For each $k \in \mathbb{Z}$ set

$$
F_{\eta, k}=\left\{x \in \mathbb{R}^{2}:\left|x_{1}\right| \leq 1 / 4,2^{k} L \leq x_{2} \leq 2^{k} L+\eta / 4\right\} .
$$

We define $k_{0}$ to be the smallest integer $k$ satisfying $2^{k} \geq \eta \max (1 / 4 L, 1 / \gamma(\epsilon))$ and note that when $k \geq k_{0}$, the sets $F_{\eta, k}$ are disjoint and we have the lower bound

$$
M f_{\eta}(x) \geq(2 \eta)^{-1 / q} \gamma^{-1}\left(2^{-k} \eta\right) \text { for } x \in F_{\eta, k}
$$

Therefore

$$
(4 \eta)^{-1} \sum_{k \geq k_{0}}\left|F_{\eta, k}\right|\left[\gamma^{-1}\left(2^{-k} \eta\right)\right]^{q} \leq \sum_{k \geq k_{0}} \int_{F_{\eta, k}} M f_{\eta}(x)^{q} d x \leq\left\|M f_{\eta}\right\|_{q}^{q} .
$$


Since $\left|F_{\eta, k}\right|=\eta / 4$ for each $k$ and $\sigma\left(\mathcal{C}\left(e_{2}, 2^{-k} \eta\right) \leq B \gamma^{-1}\left(2^{-k} \eta\right)\right.$ for some $B>0$ depending only on $\gamma$, we have

$$
\begin{aligned}
B^{-q} \int_{0}^{\delta_{1}} \sigma\left(\mathcal{C}\left(e_{2}, \delta\right)\right)^{q} \frac{d \delta}{\delta} & \leq B^{-q} \sum_{k \geq k_{0}} \int_{2^{-k-1} \eta}^{2^{-k} \eta} \sigma\left(\mathcal{C}\left(e_{2}, \delta\right)\right)^{q} \frac{d \delta}{\delta} \\
& \leq \sum_{k \geq k_{0}} 4 \eta^{-1}\left|F_{\eta, k}\right|\left[\gamma^{-1}\left(2^{-k} \eta\right)\right]^{q}
\end{aligned}
$$

for some $\delta_{1}>0$ depending only on $\gamma$. Thus from the two previous chains of inequalities we obtain $\int_{0}^{\delta_{1}} \sigma\left(\mathcal{C}\left(e_{2}, \delta\right)\right)^{q} \frac{d \delta}{\delta} \leq 8\left\|M f_{\eta}\right\|_{q}^{q}$ and this completes the proof of (6).

We now assume $q=2$ and that the condition on the caps $\mathcal{C}(\theta, \delta)$ in (5) is satisfied. By a partition of unity, the translation invariance and positivity of $\mathcal{M}$, we may suppose that the maximal operator is of the form (17). The averaging operators $A_{k}$ are convolution operators with Fourier multipliers

$$
m_{k}(\xi)=m_{0}\left(2^{k} \xi\right):=\int_{|t| \leq \epsilon} e^{-i 2^{k}\left[\xi_{1} t+L \xi_{2}-\xi_{2} \gamma(t)\right]} d t .
$$

For small $\xi$ we have the trivial bound

$$
\left|m_{k}(\xi)-2 \epsilon\right| \leq C 2^{k}|\xi|
$$

where $C$ is a universal constant. For large $|\xi|$ we will use the bound

$$
\left|m_{k}(\xi)\right| \leq C \Lambda\left(\frac{\xi}{|\xi|},\left(2^{k}|\xi|\right)^{-1}\right)
$$

which follows from (4).

Now fix a Schwartz function $\Phi$ with $\int \Phi(x) d x=2 \epsilon$ and define $\Phi_{k}(x):=$ $2^{-2 k} \Phi\left(2^{-k} x\right)$. In order to prove the $L^{2}$ boundedness of $M$, we note the pointwise bound

$$
M f(x) \leq \sup _{k \in \mathbb{Z}}\left|\Phi_{k} * f(x)\right|+\left(\sum_{k \in \mathbb{Z}}\left|A_{k} f(x)-\Phi_{k} * f(x)\right|^{2}\right)^{1 / 2} .
$$

The first term on the right hand side is dominated by the Hardy-Littlewood maximal function of $f$ and thus defines a bounded operator on all $L^{q}, q>1$. Therefore it suffices by Plancherel's theorem for the second term to show that the function

$$
\xi \rightarrow \sum_{k \in \mathbb{Z}}\left|m_{0}\left(2^{k} \xi\right)-\widehat{\Phi}\left(2^{k} \xi\right)\right|^{2}
$$

is a bounded function in $\xi$. From (18) and (19), we see that the boundedness of this function of $\xi$ will follow if we can show that

$$
I(\xi):=\sum_{k: 2^{k}|\xi| \geq C} \sigma\left(\mathcal{C}\left(\frac{\xi}{|\xi|},\left[2^{k}|\xi|\right]^{-1}\right)\right)^{2}
$$


is uniformly bounded in $\xi$ for some large $C$. Now

$$
\sigma\left(\mathcal{C}\left(\frac{\xi}{|\xi|},\left[2^{k}|\xi|\right]^{-1}\right)\right)^{2} \leq \ln 2 \int_{\left[2^{k}|\xi|\right]^{-1}}^{\left[2^{k-1}|\xi|\right]^{-1}} \sigma\left(\mathcal{C}\left(\frac{\xi}{|\xi|}, \delta\right)\right)^{2} \frac{d \delta}{\delta}
$$

and so

$$
I(\xi) \lesssim \sup _{\theta \in S^{1}} \int_{0}^{\delta_{0}} \sigma(\mathcal{C}(\theta, \delta))^{2} \frac{d \delta}{\delta}
$$

establishing the sufficiency part of Theorem 1.2 .

\section{Proof of Theorem 1.3}

We let $k_{\circ}=\min \left\{k: 2^{-k} \leq \frac{1}{4} \gamma^{\prime \prime}(1)\right\}$ and only need to consider the terms $P_{k}$ Af with $k>k_{\circ}$. Define

$$
h(t)=t^{2} \gamma^{\prime \prime}(t)
$$

so that $\gamma(t) \leq h(t)$. For $k>k_{\circ}$ we define a finite increasing sequence $\left\{t_{k, n}\right\}_{n=1}^{N_{k}}$ so that $\gamma^{\prime \prime}$ doubles on those points (as long as $t_{k, n}<1$ ) and denote the corresponding images under $\gamma^{\prime \prime}$ by $\rho_{k, n}$. We set

$$
\begin{aligned}
t_{k, 0} & =h^{-1}\left(2^{-k}\right) \\
\rho_{k, 0} & =\gamma^{\prime \prime}\left(t_{k, 0}\right),
\end{aligned}
$$

and, for $n \geq 1$, set

$$
\rho_{k, n}=\left\{\begin{array}{ll}
2^{n} \gamma^{\prime \prime}\left(t_{k, 0}\right) & \text { if } \gamma^{\prime \prime}\left(t_{k, 0}\right) \leq 2^{-n-1} \gamma^{\prime \prime}(1) \\
\gamma^{\prime \prime}(1) & \text { if } \gamma^{\prime \prime}\left(t_{k, 0}\right)>2^{-n-1} \gamma^{\prime \prime}(1)
\end{array},\right.
$$

and

$$
t_{k, n}=\gamma^{\prime \prime-1}\left(\rho_{k, n}\right)
$$

Define

$$
\begin{aligned}
& A_{k, 0} f(x)=\int_{0}^{t_{k, 0}} P_{k} f\left(x_{1}-t, x_{2}-\gamma(t)\right) d t \\
& A_{k, n} f(x)=\int_{t_{k, n-1}}^{t_{k, n}} P_{k} f\left(x_{1}-t, x_{2}-\gamma(t)\right) d t, \quad n \geq 1 .
\end{aligned}
$$

If we let

$$
N_{k}=1+\max \left\{\nu: \gamma^{\prime \prime}\left(t_{k, \nu}\right) \leq \frac{1}{2} \gamma^{\prime \prime}(1)\right\}
$$

then $t_{k, n}<1$ if $n \leq N_{k}-1$ and $t_{k, n}=1$ for $n \geq N_{k}$; consequently $A_{k, n}=0$ for $n>N_{k}$. By Minkowski's inequality we have

$$
\left\|\left(\sum_{k>k_{\circ}}\left|w_{k} P_{k} A f\right|^{2}\right)^{1 / 2}\right\|_{p} \leq \sum_{n=0}^{\infty}\left\|\left(\sum_{\substack{k: k>k_{\circ} \\ n \leq N_{k}}}\left|w_{k} A_{k, n} f\right|^{2}\right)^{1 / 2}\right\|_{p} .
$$

Theorem 1.3 follows from the following two propositions by interpolation; this can be seen as a variant of arguments in [6], [26], [35]. 
Proposition 4.1. For $n \geq 0$

$$
\left\|\left(\sum_{k \geq k_{\circ}}\left|w_{k} A_{k, n} f\right|^{2}\right)^{1 / 2}\right\|_{2} \leq C 2^{-n / 2}\|f\|_{2} .
$$

Proposition 4.2. For any $\epsilon>0$ and for $1<p<\infty$,

$$
\left\|\left(\sum_{k \geq k \circ}\left|w_{k} A_{k, n} f\right|^{2}\right)^{1 / 2}\right\|_{p} \leq C_{\epsilon} 2^{n(\epsilon+b)}\|f\|_{p}
$$

for all $n \geq 0$.

For the proof of the propositions we need to relate the numbers $t_{k, 0}$ to the weights $w_{k}$, and we get

$$
w_{k} t_{k, 0} \leq 1 \text { for } k>k_{\circ} .
$$

For $k>k_{\circ}$ the range of $h$ includes $2^{-k}$, and thus (24) follows from part (i) of the following lemma. Part (ii) shows that (24) is effective.

Lemma 4.3. Let $\gamma$ be as in Theorem 1.3.

(i) If $0 \leq s \leq \gamma(1)$ then $s \leq h(1)$ and $h^{-1}(s) \leq \gamma^{-1}(s)$.

(ii) If $0 \leq s \leq h(1 / 3)$ then $s \leq \gamma(1)$ and $\gamma^{-1}(s) \leq 3 h^{-1}(s)$.

Proof. Note that the range of $h$ and $\gamma^{\prime \prime}$ on $[0,1]$ is the same. (i) follows from $\gamma(t) \leq h(t)=t^{2} \gamma^{\prime \prime}(t)$ for $0 \leq t \leq 1$. But since $\gamma(0)=\gamma^{\prime}(0)=0$ and since $\gamma^{\prime \prime}$ is increasing we get the better inequality $\gamma(t) \leq h(t) / 2$ which is immediate from Taylor's theorem. (ii) follows from $h(t) \leq \gamma(3 t)$ for $0 \leq t \leq 1 / 3$ which holds by the monotonicity of $\gamma^{\prime}$ and $\gamma^{\prime \prime}$; indeed $t^{2} \gamma^{\prime \prime}(t) \leq t \int_{t}^{2 t} \gamma^{\prime \prime}(u) d u \leq$ $t \gamma^{\prime}(2 t) \leq \int_{2 t}^{3 t} \gamma^{\prime}(u) d u \leq \gamma(3 t)$.

We will now turn to the proof of the propositions. Proposition 4.1 relies on van der Corput's lemma (ch.VIII, §1.2. in 31]), while the proof of Proposition 4.2 relies on the eight authors' theorem [3] on the boundedness of the maximal operator along $(t, \gamma(t))$ under the $\gamma^{\prime}$ doubling hypothesis.

Proof of Proposition 4.1. We may assume $n \leq N_{k}$. Then for $n \geq 1$ we need to estimate the multiplier

$$
m_{k, n}(\xi)=\int_{t_{k, n-1}}^{t_{k, n}} \exp \left(i\left(\xi_{1} t+\xi_{2} \gamma(t)\right)\right) d t
$$

when $2^{k-1} \leq|\xi| \leq 2^{k+1}$.

For $t \geq t_{k, n-1}$ we have

$$
\gamma^{\prime \prime}(t) \geq \gamma^{\prime \prime}\left(t_{k, n-1}\right)=2^{n-1} \gamma^{\prime \prime}\left(t_{k, 0}\right)=2^{n-1} \gamma^{\prime \prime}\left(h^{-1}\left(2^{-k}\right)\right)
$$


and since $h(t)=t^{2} \gamma^{\prime \prime}(t)$,

$$
\gamma^{\prime \prime}\left(h^{-1}\left(2^{-k}\right)\right)=\frac{\left[h^{-1}\left(2^{-k}\right)\right]^{2} \gamma^{\prime \prime}\left(h^{-1}\left(2^{-k}\right)\right)}{\left[h^{-1}\left(2^{-k}\right)\right]^{2}}=\frac{2^{-k}}{\left[h^{-1}\left(2^{-k}\right)\right]^{2}}
$$

Thus if $\left|\xi_{2}\right| \geq \varepsilon\left|\xi_{1}\right|$ (i.e. $\left|\xi_{2}\right| \approx 2^{k}$ ) we get by van der Corput's Lemma

$$
\left|m_{k, n}(\xi)\right| \leq C\left|\xi_{2}\right|^{-1 / 2}\left|\gamma^{\prime \prime}\left(t_{k, n-1}\right)\right|^{-1 / 2} \leq C_{\varepsilon}|\xi|^{-1 / 2} 2^{k / 2} 2^{-\frac{n-1}{2}} h^{-1}\left(2^{-k}\right)
$$

which is at most $C_{\varepsilon}^{\prime} 2^{-n / 2} \gamma^{-1}\left(2^{-k}\right)$, by (24) .

If $\left|\xi_{2}\right| \leq \varepsilon\left|\xi_{1}\right|$ and $\varepsilon$ is sufficiently small then the derivative of the phase is $\left|\xi_{1}+\xi_{2} \gamma^{\prime}(t)\right| \geq\left|\xi_{1}\right| \approx 2^{k}$ and we obtain $\left|m_{k, n}(\xi)\right| \leq C 2^{-k}$. Now

$$
2^{-k}=h\left(t_{k, 0}\right)=2^{-k / 2} 2^{\frac{1-n}{2}} \sqrt{h\left(t_{k, n-1}\right)} \lesssim \gamma^{-1}\left(2^{-k}\right) 2^{-n / 2}
$$

since $h$ is bounded and $\gamma(t) \lesssim t^{2}$. We have now proved the estimate

$$
\left|m_{k, n}(\xi) \beta\left(2^{-k}|\xi|\right)\right| \lesssim 2^{-n / 2} \gamma^{-1}\left(2^{-k}\right)
$$

for $n>0$. For $n=0$ we need to estimate

$$
m_{k, 0}(\xi)=\int_{0}^{t_{k, 0}} \exp \left(i\left(\xi_{1} t+\xi_{2} \gamma(t)\right)\right) d t
$$

and we just use the trivial bound

$$
\left|m_{k, 0}(\xi)\right| \leq t_{k, 0}=h^{-1}\left(2^{-k}\right) \leq \gamma^{-1}\left(2^{-k}\right) .
$$

These estimates imply the asserted $L^{2}$ bound by Plancherel's theorem.

Proof of Proposition 4.2. It suffices to show

$$
\left\|\left(\sum_{k}\left|w_{k} A_{k, n} f_{k}\right|^{2}\right)^{1 / 2}\right\|_{p} \leq C_{\varepsilon, p} 2^{n(b+\varepsilon)}\left\|\left(\sum_{k}\left|f_{k}\right|^{2}\right)^{1 / 2}\right\|_{p}
$$

for $1<p<\infty$ since we can apply it with $f_{k}=L_{k} f$ where $L_{k}$ is a suitable Littlewood-Paley type localization operator with $P_{k}=P_{k} L_{k}$. By a duality argument it suffices to prove (26) for $1<p \leq 2$.

Consider the maximal function

$$
\mathfrak{M} f(x)=\sup _{0<r \leq 1} \frac{1}{r} \int_{0}^{r}\left|f\left(x_{1}-t, x_{2}-\gamma(t)\right)\right| d t .
$$

By our assumption $\gamma^{\prime \prime \prime}(t) \geq 0$ and $\gamma^{\prime}(0)=\gamma^{\prime \prime}(0)=0$, the convex function $u=\gamma^{\prime}$ satisfies the doubling condition $u(2 t) \geq 2 u(t)$ for $0 \leq t \leq 1 / 2$. Thus by [3] the maximal operator $\mathfrak{M}$ is bounded on $L^{p}\left(\mathbb{R}^{2}\right)$ for $1<p<\infty$. We also have the vector-valued version

$$
\left\|\left\{\mathfrak{M} f_{k}\right\}_{k \in \mathbb{Z}}\right\|_{L^{p}\left(\ell^{q}\right)} \lesssim\left\|\left\{f_{k}\right\}_{k \in \mathbb{Z}}\right\|_{L^{p}\left(\ell^{q}\right)}, \quad 1<p<\infty, \quad p \leq q \leq \infty .
$$

Indeed for $p=q$ this follows from the $L^{p}$ inequality for $\mathfrak{M}$ and interchanging summation and integration. For $q=\infty$ it follows from using $\sup _{k} \mathfrak{M} f_{k}=$ $\mathfrak{M}\left(\sup _{k}\left|f_{k}\right|\right)$ and applying the $L^{p}$ inequality for $\mathfrak{M}$. For $p \leq q \leq \infty$ we use 
a standard linearization and complex interpolation argument. In particular if $1<p \leq 2$, (28) holds for $q=2$.

Now note the trivial majorization

$$
\left|A_{k, n} f_{k}(x)\right| \leq t_{k, n} \mathfrak{M}\left[P_{k} f_{k}\right](x) .
$$

We shall show that given $\varepsilon>0$ in the statement of the proposition we have

$$
\sup _{\substack{k, n: \\ n \leq N_{k}}} 2^{-n(b+\varepsilon)} w_{k} t_{k, n} \leq C(\varepsilon) \text {. }
$$

Using (29) we may estimate

$$
\begin{aligned}
\left\|\left(\sum_{k}\left|w_{k} A_{k, n} f_{k}\right|^{2}\right)^{1 / 2}\right\|_{p} & \leq C(\varepsilon) 2^{n(b+\varepsilon)}\left\|\left(\sum_{k}\left|\mathfrak{M} P_{k} f_{k}\right|^{2}\right)^{1 / 2}\right\|_{p} \\
& \leq C^{\prime}(\varepsilon) 2^{n(b+\varepsilon)}\left\|\left(\sum_{k}\left|f_{k}\right|^{2}\right)^{1 / 2}\right\|_{p}
\end{aligned}
$$

by (28) , and vector-valued singular integral estimates for the operator $\left\{f_{k}\right\} \mapsto$ $\left\{P_{k} f_{k}\right\}$ on $L^{p}\left(\ell^{2}\right)$.

It remains to show (29). By (24) the required bound holds for $n=0$. Moreover

$$
w_{k} t_{k, n} \leq \frac{\gamma^{\prime \prime-1}\left(\rho_{k, n}\right)}{h^{-1}\left(2^{-k}\right)}=\frac{\gamma^{\prime \prime-1}\left(\rho_{k, n}\right)}{\gamma^{\prime \prime-1}\left(\rho_{k, 0}\right)} .
$$

Consequently it suffices to show that

$$
\sup _{\substack{k, n: \\ 1 \leq n \leq N_{k}}} 2^{-n(b+\varepsilon)} \frac{\gamma^{\prime \prime-1}\left(\rho_{k, n}\right)}{\gamma^{\prime \prime-1}\left(\rho_{k, 0}\right)} \leq C(\varepsilon),
$$

or, equivalently

$$
-b \ln 2+\frac{1}{n} \ln \left(\frac{\gamma^{\prime \prime}-1}{\gamma^{\prime \prime}\left(\rho_{k, n}\right)}\right) \leq \varepsilon \ln (2)+\frac{\ln (C(\varepsilon))}{n},
$$

for any $(k, n)$ with $k>k_{\circ}$ and $1 \leq n \leq N_{k}$. Note that $n^{-1} \ln \left(\rho_{k, n} / \rho_{k, 0}\right)=1$ for $n<N_{k}$ and $1 \leq n^{-1} \ln \left(\rho_{k, n} / \rho_{k, 0}\right) \leq \frac{n+1}{n}$ for $n=N_{k}$. The left hand side of (30) is then equal to

$$
\frac{1}{n} \int_{\rho_{k, 0}}^{\rho_{k, n}} \tau_{b}(s) \frac{d s}{s}+E_{k, n}
$$

where

$$
\tau_{b}(s)=\frac{s}{\gamma^{\prime \prime \prime}\left(\gamma^{\prime \prime-1}(s)\right) \gamma^{\prime \prime-1}(s)}-b,
$$

$E_{k, n}=0$ for $n<N_{k}$ and $\left|E_{k, n}\right| \leq n^{-1}$ for $n=N_{k}$. Thus it suffices to show that there is $\mathcal{N}(\varepsilon)>0$ so that

$$
\sup _{k: N_{k}>n} \frac{1}{n} \int_{\rho_{k, 0}}^{\rho_{k, n}}\left|\tau_{b}(s)\right| \frac{d s}{s} \leq \varepsilon \ln (2) \text { for } n>\mathcal{N}(\varepsilon) .
$$


Now $\gamma^{\prime \prime}-1(s) \rightarrow 0$ as $s \rightarrow 0$ and, since $b=\lim _{t \rightarrow 0} \frac{\gamma^{\prime \prime}(t)}{t \gamma^{\prime \prime \prime}(t)}$, we have therefore $\lim _{s \rightarrow 0} \tau_{b}(s)=0$. Choose $\delta(\varepsilon) \in\left(0, \frac{1}{2} \gamma^{\prime \prime}(1)\right)$ so that $\left|\tau_{b}(s)\right|<\varepsilon / 4$ whenever $s<\delta(\varepsilon)$. Because of the assumed behavior of $\frac{\gamma^{\prime \prime}(t)}{t \gamma^{\prime \prime \prime}(t)}$ near 0 we see that $M=\sup _{0<t \leq 1} \frac{\gamma^{\prime \prime}(t)}{t \gamma^{\prime \prime \prime}(t)}<\infty$. Let $\mathcal{N}(\varepsilon)=4 M \varepsilon^{-1} \ln \left(\frac{\gamma^{\prime \prime}(1)}{\delta(\varepsilon)}\right)$.

Let $I_{k, n}=\left[\rho_{k, 0}, \rho_{k, n}\right] \cap(0, \delta(\varepsilon)]$ and $J_{k, n}=\left[\rho_{k, 0}, \rho_{k, n}\right] \cap\left[\delta(\varepsilon), \gamma^{\prime \prime}(1)\right]$; one of these intervals may be empty. Now $\tau_{b}(s) \leq \varepsilon / 4$ on $I_{k, n}$ and $\rho_{k, n} / \rho_{k, 0}<2^{n+1}$. Therefore for all $n \leq N_{k}$

$$
\frac{1}{n} \int_{I_{k, n}}\left|\tau_{b}(s)\right| \frac{d s}{s} \leq \frac{\varepsilon}{4 n} \int_{\rho_{k, 0}}^{\rho_{k, n}} \frac{d s}{s} \leq \frac{n+1}{n} \frac{\varepsilon \ln 2}{4} \leq \frac{\varepsilon \ln (2)}{2} .
$$

On $J_{k, n}$ we use the estimate $\left|\tau_{b}(s)\right| \leq 2 M$ and obtain for $\mathcal{N}(\varepsilon) \leq n \leq N_{k}$

$$
\frac{1}{n} \int_{J_{k, n}}\left|\tau_{b}(s)\right| \frac{d s}{s} \leq \frac{1}{n} \int_{\delta(\varepsilon)}^{\gamma^{\prime \prime}(1)} 2 M \frac{d s}{s}=\frac{2 M \ln \left(\frac{\gamma^{\prime \prime}(1)}{\delta(\varepsilon)}\right)}{\mathcal{N}(\varepsilon)} \leq \frac{\varepsilon}{2}
$$

We combine these two estimates and obtain (31). Thus the proposition is proved.

\section{ANother open PROBLEM}

We recall another open problem concerning a lacunary maximal operator generated by dilates of a Marcinkiewicz multiplier in two dimensions. Let $\eta_{0}$ be a Schwartz function on the real line with $\eta_{0}(0) \neq 0$. Let

$$
m_{k}\left(\xi_{1}, \xi_{2}\right)=\eta_{0}\left(2^{2 k} \xi_{1} \xi_{2}\right)
$$

(the dilates of the so-called hyperbolic cross multiplier) and define

$$
\mathbb{M} f(x)=\sup _{k}\left|\mathcal{F}^{-1}\left[m_{k} \widehat{f}\right](x)\right| \text {. }
$$

Problem. Is $\mathbb{M}$ bounded on $L^{p}\left(\mathbb{R}^{2}\right)$, for some $p \in(1, \infty)$ ?

The problem is closely related to one formulated in 10 and 8 , on the pointwise convergence for the "hyperbolic" Riesz means $R_{\lambda, t} f$. These are defined by $\widehat{R_{\lambda, t} f}(\xi)=\left(1-t^{-2} \xi_{1}^{2} \xi_{2}^{2}\right)_{+}^{\lambda} \widehat{f}$ and they were studied in [10] and 2]. A positive answer to our question is known to imply positive $L^{p}\left(\mathbb{R}^{2}\right)$ boundedness results for the maximal function $M_{\lambda} f(x)=\sup _{t>0}\left|R_{\lambda, t} f(x)\right|$, for suitable $\lambda$. A negative answer would prove that $M_{\lambda}$ is unbounded on all $L^{p}\left(\mathbb{R}^{2}\right)$, for all $\lambda$.

Note that the multipliers $m_{k}$ satisfy the hypotheses of the Marcinkiewicz multiplier theorem in $\mathbb{R}^{2}$. As proved in [7] $L^{p}$ boundedness for lacunary maximal functions generated by Mikhlin-Hörmander (or Marcinkiewicz) multipliers fails generically, with respect to the topology in some natural symbol spaces. This however does not settle our question above. More results and 
open problems on minimal decay assumptions for the boundedness of such maximal operators can be found in [12].

One can also ask for bounds on the $L^{p}$ operator norm of the maximal

operator $\mathbb{M}_{N} f(x)=\sup _{|k| \leq N}\left|\mathcal{F}^{-1}\left[m_{k} \widehat{f}\right](x)\right|$. Petr Honzík [17] noted that one can improve the trivial upper bound $C_{p} N^{1 / p}$ by combining the good $\lambda$ inequalities in 20] with the reasoning in [12]; this yields the bound $C_{p} \log (N)$ (at least for a discrete analogue).

\section{REFERENCES}

[1] J. Bruna, A. Nagel, S. Wainger, Convex hypersurfaces and Fourier transforms. Ann. of Math. (2) 127 (1988), no. 2, 333-365.

[2] A. Carbery, A note on the "hyperbolic" Bochner-Riesz means. Proc. Amer. Math. Soc. 92 (1984), no. 3, 397-400.

[3] H. Carlsson, M. Christ, A. Córdoba, J. Duoandikoetxea, J.L. Rubio de Francia, J. Vance, S. Wainger, D. Weinberg, $L^{p}$ estimates for maximal functions and Hilbert transforms along flat convex curves in $R^{2}$. Bull. Amer. Math. Soc. (N.S.) 14 (1986), no. 2, 263-267.

[4] S.Y.A. Chang, R. Fefferman, A continuous version of duality of $H^{1}$ and BMO on the bidisc. Annals of Math. 112 (1980), 179-201.

[5] M. Christ, Weak type (1,1) bounds for rough operators. Annals of Math. 128 (1988), $19-42$.

[6] Failure of an endpoint estimate for integrals along curves. Fourier analysis and partial differential equations (Miraflores de la Sierra, 1992), 163-168, Stud. Adv. Math., CRC, Boca Raton, FL, 1995.

[7] M. Christ, L. Grafakos, P. Honzík, A. Seeger, Maximal functions associated with Fourier multipliers of Mikhlin-Hörmander type. Math. Z. 249 (2005), no. 1, 223-240.

[8] H. Dappa, W. Trebels, On maximal functions generated by Fourier multipliers. Ark. Mat. 23 (1985), no. 2, 241-259.

[9] J. Duoandikoetxea, J. Rubio de Francia, Maximal and singular integral operators via Fourier transform estimates. Invent. Math. 84 (1986), no. 3, 541-561.

[10] A. El Kohen, On the hyperbolic Riesz means. Proc. Amer. Math. Soc. 89 (1983), no. 1, 113-116.

[11] C. Fefferman, E.M. Stein, Some maximal inequalities. Amer. J. Math. 93 (1971), $107-115$.

[12] L. Grafakos, P. Honzík, A. Seeger On maximal functions for Mikhlin-Hörmander multipliers. Adv. Math. 204 (2006), no. 2, 363-378.

[13] Y. Heo, An endpoint estimate for some maximal operators associated to submanifolds of low codimension. Pacific J. Math. 201 (2001), no. 2, 323-338.

[14] Weak type estimates for some maximal operators on Hardy spaces. Math. Nachr. 280 (2007), no. 3, 281-289.

[15] _ Endpoint estimates for some maximal operators associated to the circular conical surface. J. Math. Anal. Appl. 351 (2009), no. 1, 152-162.

[16] Y. Heo, F. Nazarov, A. Seeger, Radial Fourier multipliers in high dimensions. To appear in Acta Math.

[17] P. Honzík, Personal communication.

[18] D. M. Oberlin, An endpoint estimate for some maximal operators. Rev. Mat. Iberoamerica 12, No. 3, 641-652 (1996).

[19] J. Peetre, On spaces of Triebel-Lizorkin type. Ark. Mat. 13 (1975), 123-130.

[20] J. Pipher, Bounded double square functions. Ann. Inst. Fourier (Grenoble) 36 (1986), no. $2,69-82$. 
[21] A.N. Podkorytov, On the asymptotics of the Fourier transform on a convex curve. (Russian) Vestnik Leningrad. Univ. Mat. Mekh. Astronom. 1991, , vyp. 2, 50-57, 125; translation in Vestnik Leningrad Univ. Math. 24 (1991), no. 2, 57-65.

[22] What is it possible to say about an asymptotic of the Fourier transform of the characteristic function of a two-dimensional convex body with nonsmooth boundary? Fourier analysis and convexity, 209-215, Appl. Numer. Harmon. Anal., Birkhuser Boston, Boston, MA, 2004.

[23] M. Pramanik, K. Rogers and and A. Seeger, A Calderón-Zygmund estimate with applications to generalized Radon transforms and Fourier integral operators. Studia Mathematica, to appear.

[24] M. Pramanik and A. Seeger, $L^{p}$ regularity of averages over curves and bounds for associated maximal operators. Amer. J. Math. 129 (2007), no. 1, 61-103.

[25] A. Seeger, Remarks on singular convolution operators. Studia Math. 97 (1990), 91114.

[26] _ Degenerate Fourier integral operators in the plane. Duke Math. J. 71 (1993), no. $3,685-745$.

[27] A. Seeger and T. Tao, Sharp Lorentz space estimates for rough operators, Math. Ann. 320 (2001), 381-415.

[28] A. Seeger, T. Tao and J. Wright, Endpoint mapping properties of spherical maximal operators. Journal de l'Institut Mathématiques de Jussieu, 2 (2003), 109-144.

[29] _ Singular maximal functions and Radon transforms near $L^{1}$. Amer. J. Math. 126 (2004), no. 3, 607-647. See also http://www.math.wisc.edu/ seeger/papers/corr-stw-amj.pdf for a correction.

[30] C.D. Sogge, E. M. Stein, Averages over hypersurfaces. Smoothness of generalized Radon transforms. J. Analyse Math. 54 (1990), 165-188.

[31] E.M. Stein, Harmonic analysis: real-variable methods, orthogonality, and oscillatory integrals. Princeton Mathematical Series, 43. Monographs in Harmonic Analysis, III. Princeton University Press, Princeton, NJ, 1993.

[32] E.M. Stein, S. Wainger, Problems in harmonic analysis related to curvature. Bull. Amer. Math. Soc. 84 (1978), no. 6, 1239-1295.

[33] T. Tao, The $H^{1} \rightarrow L^{1, \infty}$ boundedness of the cylindrical maximal function, unpublished notes 2002.

[34] T. Wolff, Local smoothing type estimates on $L^{p}$ for large p. Geom. Funct. Anal. 10 (2000), no. 5, 1237-1288.

[35] C.W. Yang, $L^{p}$ regularity of averaging operators with higher fold singularities. Proc. Amer. Math. Soc. 131 (2003), no. 2, 455-465.

Department of Mathematics, University of Wisconsin-Madison, 480 Lincoln Drive, MADison, WI 53706, USA

E-mail address: seeger@math.wisc.edu

Maxwell Institute for Mathematical Sciences and the School of Mathematics, University of Edinburgh, JCMB, King's Buildings, Mayfield Road,, Edinburgh EH9 3JZ, ScOtLAND

E-mail address: J.R.Wright@ed.ac.uk 